\title{
Fabrication and characterizations of hydrogels for cartilage repair
}

\begin{abstract}
Articular cartilage is a vascular tissue with limited repair capabilities, leaving an afflicted person in extreme pain. The tissue experiences numerous forces throughout its lifetime. This study focuses on development of a novel hydrogel composed of chitosan and $\beta$-glycerophosphate for articular cartilage repair. The aim of this study was to investigate the mechanical properties and swelling behaviour of a novel hydrogel composed of chitosan and $\beta$-glycerophosphate for cartilage repair. The mechanical properties were measured for compression forces. Mach-1 mechanical testing system was used to obtain storage and loss modulus for each hydrogel sample to achieve viscoelastic properties of fabricated hydrogels. Two swelling tests were carried out to compare water retaining capabilities of the samples. The hydrogel samples were made of five different concentrations of $\beta$-glycerophosphate cross-linked with chitosan. Each sample with different $\beta$-glycerophosphate concentration underwent sinusoidal compression forces at three different frequencies $-0.1 \mathrm{~Hz}, 0.316 \mathrm{~Hz}$ and $1 \mathrm{~Hz}$. The result of mechanical testing was obtained as storage and loss modulus. Storage modulus represents the elastic component and loss modulus represents the viscosity of the samples. The results obtained for $1 \mathrm{~Hz}$ were of interest because the knee experiences frequency of $1 \mathrm{~Hz}$ during walking.
\end{abstract}

Keywords: articular cartilage, chitosan, cross-linker, hydrogel, loss modulus, scaffold, storage modulus, swelling property, viscoelastic
Volume 2 Issue 6 - 2017

\author{
Payal Kaur,' Seyed Ali Khaghani,' Agbabiaka \\ Oluwadamilola,' Zohaib Khurshid, ${ }^{2}$ \\ Muhammad Sohail Zafar, ${ }^{3}$ Masoud \\ Mozafari, 4,5 Mansour Youseffi,' Farshid Sefat I \\ 'Department of Medical Engineering, University of Bradford, UK \\ ${ }^{2}$ Department of Dental Biomaterials, King Faisal University, \\ Saudi Arabia \\ ${ }^{3}$ Department of Restorative Dentistry, Taibah University, Saudi \\ Arabia \\ ${ }^{4}$ Nanotechnology and Advanced Materials Department, \\ Materials and Energy Research Center (MERC), Iran \\ ${ }^{5}$ Department of Tissue Engineering \& Regenerative Medicine, \\ Iran University of Medical Sciences, Iran
}

Correspondence: Farshid Sefat, School of Engineering, Medical Engineering Department, University of Bradford, Bradford, West Yorkshire, BD7 IDP, UK, Tel (+)44-1274233679,

Email F.Sefatl@Bradford.ac.uk

Received: March 28, 2017 | Published: September 26, 2017
Abbreviations: AA, acetic acid; E', storage modulus; E", loss modulus; ECM, extracellular matrix; GAGs, glycos amino glycans; $\mathrm{M}$, molar; $\mathrm{NaOH}$, sodium hydroxide; PBS, phosphate buffer solution; PCM, pericellular matrix; $\beta$-GP, beta-glycerophosphate

\section{Introduction}

Several polymers are being used in the medical and pharmaceutical fields for various purposes such as accurate drug delivery, tissue repair, suturing and many more. Natural polysaccharides are highly versatile polymeric materials. ${ }^{1}$ They are proven to have many biomedical applications from their ability in living cell encapsulation, bone and cartilage repair to the making of biocompatible scaffolds used for tissue regeneration. The properties of polysaccharides are related to the chemical composition of the macromolecules they consist of. These properties can be tailored depending on the intended use. Polysaccharides are found in abundant quantities, for example, cellulose is known to be the most abundant organic component found on earth. They are available from several natural sources such as animals in the forms of chitin and chondroitin; from plants in the forms of pectin and guar gum; algae in the forms of alginates and agar; fungi in the forms of xanthan gum and gellan. Polysaccharides have been used as food additives and have applications in cosmetic products. ${ }^{1}$

Polysaccharides are considered safe because they are nontoxic, biodegradable and biocompatible. Polysaccharides can form three dimensional networks such as hydrogels which can retain large amounts of biological fluids or water. This function is very similar to the natural tissues which leading to more applications of polysaccharides when they are used for formation of hydrogels. The structure of such hydrogels can depend on the type of cross linker used to change the property of its macromolecules, depending on its application; for example, bone regeneration requires a different crosslinker than cartilage regeneration. ${ }^{1}$

In the broad field of tissue engineering and regenerative medicine, scaffolds enable us to obtain functional living implants from different cell cultures. Polysaccharides have been studied a lot to achieve desired shapes which are modelled based on specific needs while being able to host cells. Regeneration of bone and cartilage would not only require appropriate polymeric materials but growth factors such as IGF, FGF and TGF- $\beta$ are also essential.

Hydrogels have not been given an exact definition. They are commonly described as three dimensional water swollen polymeric materials with significant swelling properties. They have received a lot of attention due to their potential for biomedical applications. ${ }^{2}$ The key properties for the hydrogels include their hydrophilic structure allowing them to hold large amounts of water and their everlasting service. This three dimensional network is insoluble in water at physiologic $\mathrm{pH}$, ionic strength and temperature. The predominant medium of hydrogel is water, held together by covalent cross links, hydrogen bonds, ionic bonds, hydrophobic interactions, physical bonds and bio-recognition interactions. ${ }^{2,3}$ Physical gels tend to show similar behaviour as covalent gels in which there are isolated bonds linking the gels together physically. The isolated bonds present are weak because they are hydrogen bonds. The weak bonds are present in a different sequence for each gel and the difference in their physical and covalent bonds shows in the way each responds to different temperature. Close attention should be paid to the physical interactions between the gels as this plays an important role. ${ }^{2}$ The properties of the polymer used and the density of network joints have an effect on the hydrogel's formation and behaviour. Hydrogels in equilibrium or in their swollen state contain a large amount of water. In swollen state, the hydrogel holds a greater mass fraction of water 
than the mass fraction of the polymer itself. ${ }^{4}$ In order to achieve a high level of swelling, synthetic polymers which are water soluble in non-cross-linked form are commonly used. There are several ways to synthesis the hydrogels. Parallel cross linking, polymerization of multifunctional monomers, subsequent cross-linking with reactive groups and using cross-linker to react with polymer are some of the ways to synthesis the hydrogels. ${ }^{5-8}$

There are several techniques to form the hydrogels. One of the most common techniques is to use the free radical cross-linking copolymerization of a hydrophilic neutrally charged monomer with a small quantity of cross-linker. In the reaction mixture, addition of ionic co-monomer enhances the swelling capacity of the hydrogel. This is carried out in aqueous solution because the monomers tend to be solid at polymerization temperature. For a swollen hydrogel, the water is bound to the polymer chain by hydrophobic or polar interactions. Within a hydrogel there are some unbound water molecules present which allows the solutes to diffuse through. According to Flory et al, the degree to which a cross-linked hydrogel would swell is directed by the thermodynamic compatibility of polymer and elastic forces present in polymer chains. ${ }^{9}$

Some of the hydrogels are temperature sensitive. This affects the swelling and de-swelling properties of the hydrogel due to the swelling agent being affected by the change in temperature which may be outside its compatibility range. The term LCST-lower critical solution temperature is used to describe this behaviour of the hydrogels. A temperature sensitive hydrogel is soluble below LCST. Above the LCST, the hydrogel may response drastically. The extreme change to its environment may cause it to collapse. When the gel is placed below LCST, it re-swells as a result of increased hydrophobic bonding with water. ${ }^{2,10}$

\section{Materials and methods}

From background research, it was known that chitosan can only be dissolved in weak acid with the pH below 6 at the concentration of $0.1 \mathrm{M} .{ }^{11}$ Chitosan provided by Sigma Aldrich was in a solid form (powder) and it was dissolved in an aqueous solution of acid to make it soluble for making a hydrogel. ${ }^{11}$

\section{Preparation of acetic acid (AA)}

In order to prepare chitosan $0.1 \mathrm{M}$ of acetic acid was used. First step was to prepare acetic acid with $0.1 \mathrm{M}$ from $1 \mathrm{M}$ which was purchased from Sigma Aldrich. This meant that there would be 1\% acetic acid in $100 \mathrm{ml}$ solution $(1 \mathrm{ml}$ acetic acid in $99 \mathrm{ml}$ of deionised water). To prepare this, $0.3 \mathrm{ml}$ of acetic acid of $17.416 \mathrm{M}$ concentration was added to $47.7 \mathrm{ml}$ of deionised water, making a $50 \mathrm{ml}$ solution of $0.3483 \mathrm{M}$ acetic acid.

\section{Preparation of chitosan solution}

Chitosan (Sigma Aldrich, UK) solution with acetic acid was prepared in the ratio of 1:49 (1g of chitosan was dissolved in $49 \mathrm{ml}$ of acetic acid $(0.34832 \mathrm{M}))$. The mixture was stirred at a fast pace before adding more chitosan powder ( $0.2 \mathrm{~g}$ each time). After adding $1 \mathrm{~g}$ of chitosan, the mixture was left to stir for 90 minutes on magnetic stirring plate. Hydrogel was formed after 90 minutes with too many air bubbles and therefore, it was left to rest for 60 minutes to reduce the air bubbles. Once hydrogel had degassed, the mixture was split evenly in 10 small beakers $(2 \mathrm{ml}$ each) in order to cross-linker to be added.

\section{Preparation of $\beta$-glycerophosphate disodium solution}

The $\beta$-Glycerophosphate disodium salt was prepared to be added to chitosan hydrogel. The ratio for $\beta$-Glycerophosphate disodium salt and deionised water used was $500 \mathrm{mg}$ per $\mathrm{ml}$. The mixture of both was stirred on magnetic stirrer for 30 minutes using a magnetic stirring bar.

\section{Chitosan- $\beta$-glycerophosphate hydrogel preparation}

Once the chitosan hydrogel and $\beta$-Glycerophosphate disodium salt solution were prepared, they had to be mixed together for crosslinking purposes. The prepared $\beta$-Glycerophosphate was added to the chitosan solutions (dropwise) at different concentrations ranging from $5 \mathrm{mg} / \mathrm{ml}$, $10 \mathrm{mg} / \mathrm{ml}, 25 \mathrm{mg} / \mathrm{ml}, 50 \mathrm{mg} / \mathrm{ml}$ and $100 \mathrm{mg} / \mathrm{ml}$.

The mixtures were stirred for a minimum of 30 minutes. The prepared gel was left to degas for 3 hours at room temperature. Once the gel had degassed, the prepared chitosan/ $\beta$-GP hydrogel was placed in tubes and the ends were covered with biofilm. This was done to provide them with cylindrical shape. The hydrogels were left at room temperature for 24 hours. The tubes were then places at $-80^{\circ} \mathrm{C}$ as hydrogel will not crystallise at this low temperature. The samples were neutralised by $0.5 \mathrm{M}$ Sodium hydroxide $(\mathrm{NaOH})$. The samples were individually soaked in $\mathrm{NaOH}$. In order to make sure complete neutralization occurred, samples were placed against bright background to check whether it has darker mass in the center. Samples were left within $\mathrm{NaOH}$ for longer period if darker mass-like structure was present. The following five samples were prepared as shown in Table 1.

Table I Five different samples prepared with addition of Chitosan/Acetic acid to the beta/Glycerophosphate cross linker.

\begin{tabular}{|c|c|c|c|}
\hline Sample & Chitosan/acetic acid & Beta-glycerophosphate $(\mathrm{mg} / \mathrm{ml})$ & $\begin{array}{l}\text { Chitosan beta- } \\
\text { glycerophosphate }(\mathrm{ml})\end{array}$ \\
\hline $\mathrm{V}$ & $2 \mathrm{~g} / \mathrm{ml} \mathrm{CS} / \mathrm{AA}$ & 0.01 & $0.02 \mathrm{ml} \beta-\mathrm{GP}$ \\
\hline W & $2 \mathrm{~g} / \mathrm{ml} \mathrm{CS} / \mathrm{AA}$ & 0.02 & $0.04 \mathrm{ml} \beta-\mathrm{GP}$ \\
\hline$x$ & $2 \mathrm{~g} / \mathrm{ml} \mathrm{CS} / \mathrm{AA}$ & 0.05 & $0.10 \mathrm{ml} \beta-\mathrm{GP}$ \\
\hline Y & $2 \mathrm{~g} / \mathrm{ml} \mathrm{CS} / \mathrm{AA}$ & 0.1 & $0.20 \mathrm{ml} \beta-\mathrm{GP}$ \\
\hline Z & $2 \mathrm{~g} / \mathrm{ml} \mathrm{CS} / \mathrm{AA}$ & 0.2 & $0.40 \mathrm{ml} \beta$-GP \\
\hline
\end{tabular}

\section{Mechanical test}

Compression test with various frequencies was carried out to determine the viscoelasticity behavior of hydrogels. During normal walking, the body's center of mass and vertical projection into spatial trajectory results in a sinusoidal pattern. This is due to the shift in weight from one leg to another during normal walk. ${ }^{2}$ For this reason, compression test in sinusoid waveform loading mode was carried out on all samples. The viscoelastic properties were characterised by the storage (E') and loss (E") moduli of a material. Storage modulus 
is the material's ability to store energy for elastic recoil after stress is removed, representing elastic response. The loss modulus is the material's ability to dissipate energy, representing viscous response. ${ }^{13}$ Polymer modulus is temperature and time dependent.

\section{Statistical analysis}

One way ANOVA was carried out for this study. The null hypothesis (H0) for the calculated data is that there is no significant variance between the E' values of different values i.e. $\mathrm{H} 0 ; \mathrm{MV}=\mathrm{MW}=\mathrm{MX}=\mathrm{MY}=\mathrm{MZ}$, where $\mathrm{MV}$ is the mean value for E' calculated for sample V. The alpha level is 0.05 which is most commonly chosen. The alternative hypothesis is that there is at least one difference between the variance of mean for E' values of samples.

\section{Results and discussion}

Mechanical analysis of five samples $-\mathrm{V}, \mathrm{W}, \mathrm{X}, \mathrm{Y}$ and $\mathrm{Z}$ was carried out at three different sinusoid frequencies of $0.1 \mathrm{~Hz}, 0.316 \mathrm{~Hz}$ and $1 \mathrm{~Hz}$. The reason for these chosen number for frequencies is that at the logarithmic scale, the midpoint for 0.1 and 1 was 0.316 instead of 0.55 . The compression test was repeated three times for each sample. The test was conducted at room temperature as biological systems remain at a constant temperature. The storage and loss moduli obtained were converted to correct units with the use of height and diameter from each sample. The mean values of each frequency were used in the final plotting of the graph. The error bars on a graph were calculated using the standard deviation formula below:

$$
\sigma=\sqrt{\frac{\sum\left(x_{i}-\bar{x}\right)^{2}}{n-1}}
$$

In the equation, $\sigma$ represents standard deviation; $\sum$ represents sum of; $x_{i}$ represents each value in data; $\bar{x}$ represents the mean of values in data; $n$ represents the number of value in data.

The conversion of raw data for individual sample was used to obtain the average of each repeat at a given frequency to plot the graphs for E' and E" against the applied frequencies. Storage modulus shows elastic component of material which is in phase with applied stress (Figures 1) (Figure 2). The $\mathrm{P}$ value for the test is less than 0.05 which concludes the null hypothesis will be rejected. The null hypothesis states that there is no significance variance between the E' values of samples. Hence, the alternative hypothesis true as there is significant variance between the means for all samples.

The focus was on measurements carried out at $1 \mathrm{~Hz}$ because articular cartilage experiences this frequency during walking. The graph in Figure 2 displays the comparison of storage modulus for all samples at $1 \mathrm{~Hz}$. Sample $\mathrm{Y}$ had the highest value for storage modulus at $1 \mathrm{~Hz}$ and sample $\mathrm{W}$ had the lowest. This was due to the composition of each sample. The graph below confirms that the concentration of $\beta$-Glycerophosphate in a chitosan hydrogel has positive effect on the performance of the scaffold by altering its mechanical properties. For all samples, the values are getting smaller as the strain rate is increased which demonstrates the material is behaving elastically (Figure 3).

It is very important to test the $\mathrm{pH}$ of each sample as hydrogels are $\mathrm{pH}$ sensitive. The intention of this project was to apply the knowledge of tissue engineering to aid articular cartilage regeneration. If the biological tissue comes in contact with hydrogel of incorrect $\mathrm{pH}$, it would have a significant impact as the cells would begin to denature and surrounding tissues would be damaged. All samples were very close to the physiological $\mathrm{pH}$ of 7.04. The gel had $\mathrm{pH}$ lower than 6 prior to being neutralised by $\mathrm{NaOH}$. Sample V with $0.02 \mathrm{ml}$ of $\beta$-GP had the most suitable $\mathrm{pH}$ compare to other samples. It is possible to lower $\mathrm{pH}$ by rinsing samples with different concentration of $\mathrm{NaOH}$, However, it is suggested not to leave the samples in $\mathrm{NaOH}$ for long period as it affects the viscosity of the sample..$^{11,14}$

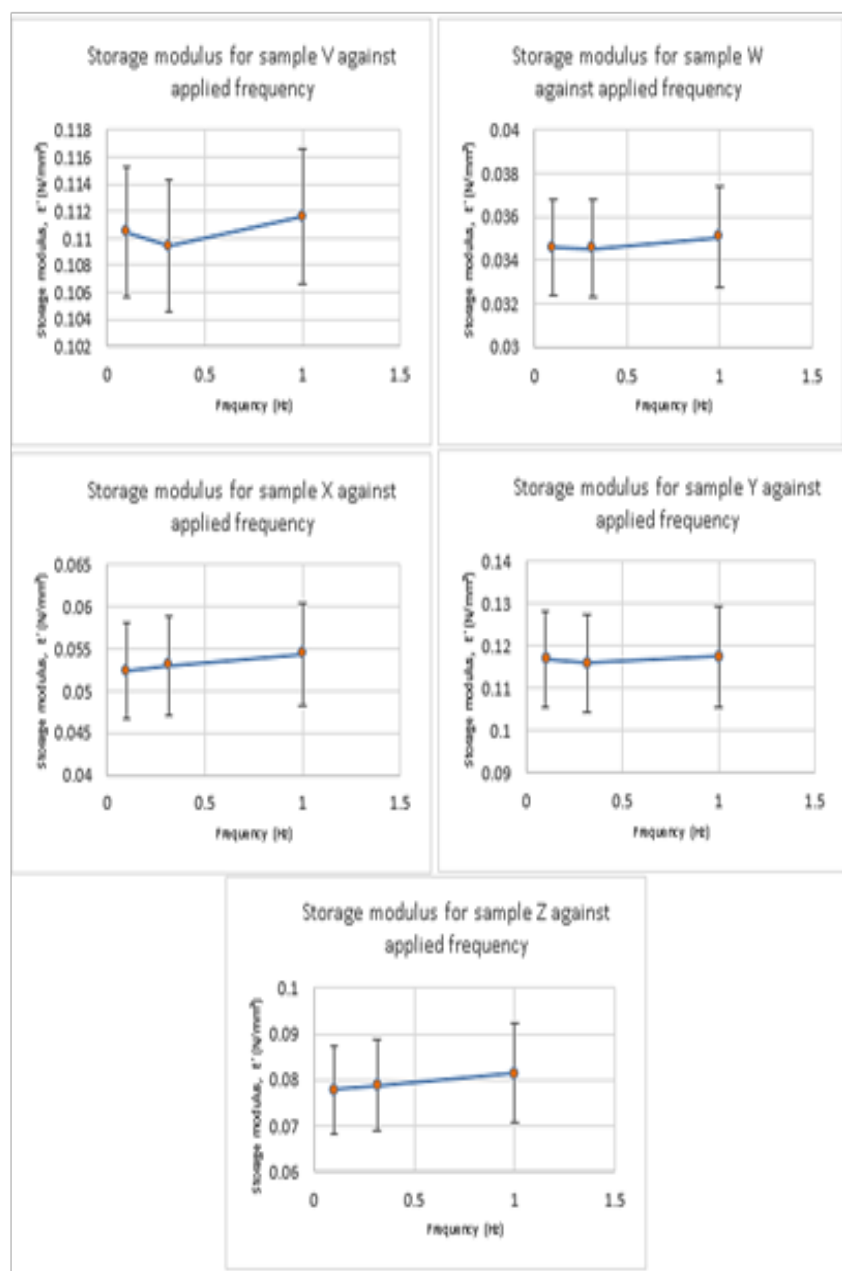

Figure I The individual graphs plotted for storage moduli (E') for each sample demonstrate that all the samples had viscoelastic properties. As the strain rate and frequencies are increased, the sample behaved more elastically. This is evident in the graphs as the storage modulus is increasing for all samples exhibiting elastic properties of the material.

Swelling test was carried out to check the swelling capacity of the chitosan hydrogels. Checking the swelling capacity of the hydrogel is very important especially if they are intended to be placed in physiological setting. ${ }^{15,16}$ Also, this test shows how the hydrogel would behave in presence of body fluids as its properties and size would change. For any given hydrogel, it is essential for them to have swelling capability to allow nutrients to diffuse through. ${ }^{17,18}$ Articular cartilage is surrounded by synovial fluid therefore it is important to see the behaviour of the formed hydrogel in the presence of fluid. ${ }^{19,20}$

The samples were immersed in PBS (Phosphate Buffer Solution) as PBS has similar viscosity as body fluids (Figure 4). It can be observed that all samples demonstrated great swelling properties 
during the first 3 hours. Another swelling test carried out (Figure 5) where all the dried samples were placed in the incubator at $37^{\circ} \mathrm{C}$ for 48 hours prior to being immersed in PBS where the volume of each sample was measured between each time periods (Table 2).

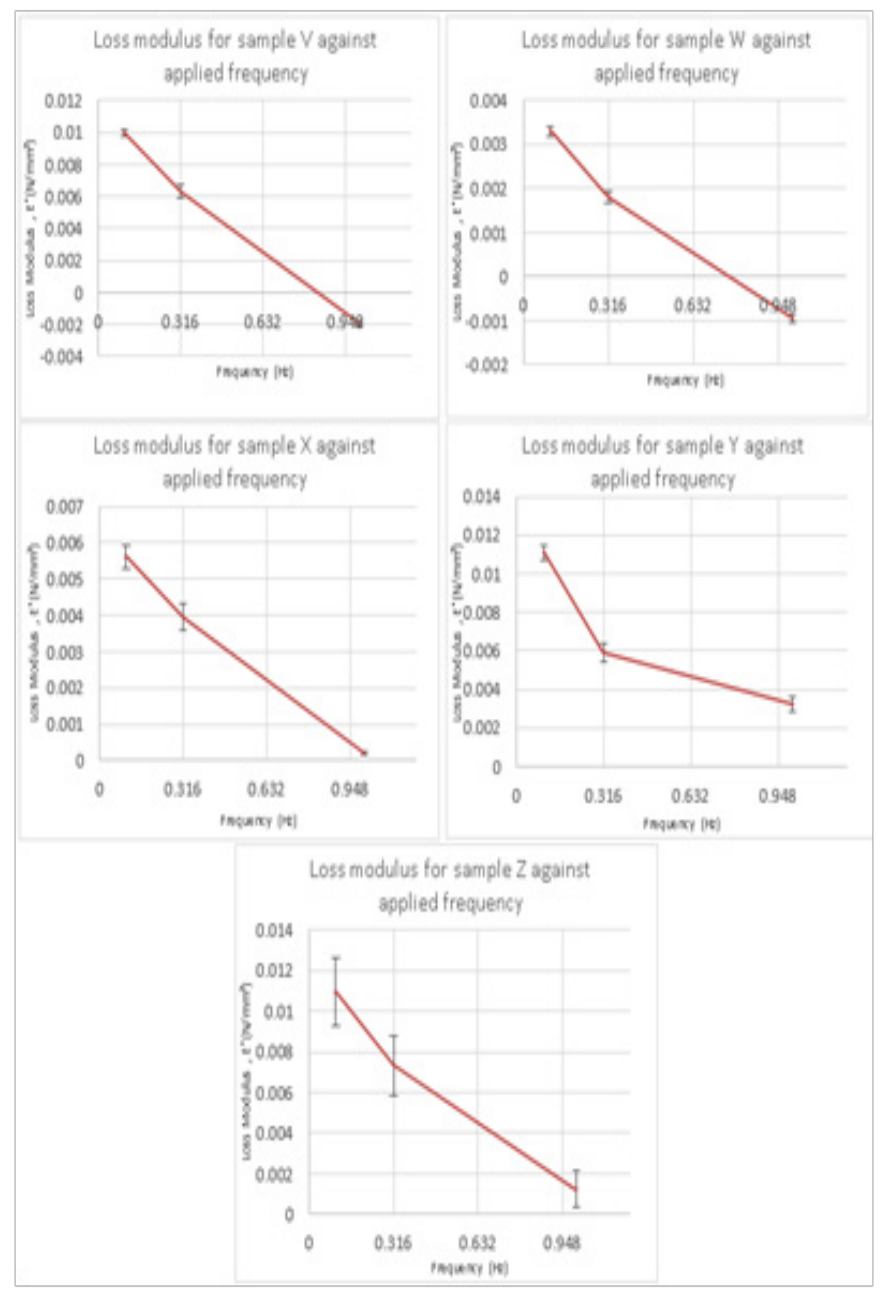

Figure 2 The individual graphs plotted for loss moduli (E') for each sample demonstrate that all the samples had viscoelastic properties. The loss modulus is decreasing for all samples as the compression force is increased displaying that all samples have viscous properties. This is the significant characteristic of articular cartilage because its viscoelastic properties not only allow the tissue to withstand shock but also allow the tissue to diffuse minerals and nutrients during loading and unloading processes.

Dried samples were immersed in PBS solution at physiological temperature of $37^{\circ} \mathrm{C}$ in an incubator. The volume was measured for all samples at 24, 48, 72, 144 and 192 hours as shown in Table 2. Sample $\mathrm{X}$ demonstrated poor swelling ability compared to all the other samples (Figure 5). From 48 to 72 hours, sample W and Y showed poor swelling ability. Sample W and Y showed similar behaviour for first 72 hours. Sample Y remained at a constant volume from 72 hours till 144 hours. Samples V, Y and Z all increased after 144 hours whereas samples $\mathrm{W}$ and $\mathrm{X}$ both decreased after 144 hours. For some samples, the water uptake seemed to be dependent on the chitosan proportion as the water uptake decreased with higher concentration of $\beta$-GP. Samples V and W showed to be less viscous compared to the other samples as they both demonstrated poor swelling capabilities.
One of the variables affecting the volume is the amount of PBS added to each sample. For samples to achieve excellent swelling properties during the first 24hours, more PBS should have been added to their beaker. Another limitation of the test was the accuracy of measuring the volume. The methodology for swelling test 2 was not precise as it did all measurements in $\mathrm{ml}$ or $\mathrm{cm}^{3}$. The measuring units should have been more precise for accurate measurement. That would have also ruled out human error to a certain extent. Also, the test is only accurate for big samples whereas the samples for this study were too small for such a method to be used. There is some question about the reasons for the fluctuation in the samples' behaviour in the incubator; this may have caused the samples to shrink rather than swell once the PBS was fully absorbed. Errors were expected in this test because it is not a precise measurement. The test was still carried out to see the behaviour of the hydrogels when immersed in fluid with similar density as biological fluid.

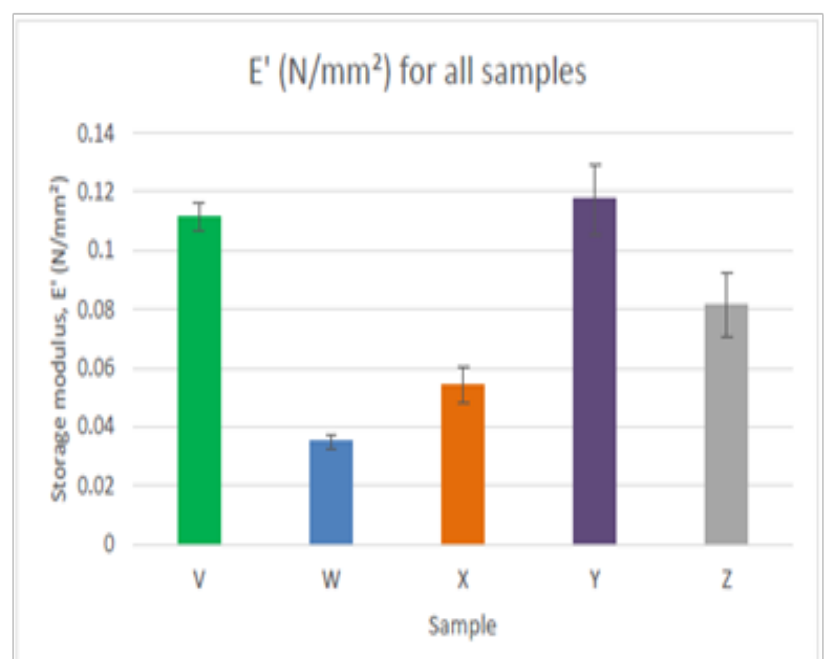

Figure 3 Comparison of storage modulus for all samples at $\mathrm{IHz}$. Sample $\mathrm{Y}$ had the highest value for storage modulus. SampleY consisted of $0.20 \mathrm{mg} / \mathrm{ml}$ of $\beta$-Glycerophosphate whereas sample $W$ which had the lowest value of storage modulus consisted of $0.04 \mathrm{mg} / \mathrm{ml} \beta$-Glycerophosphate.

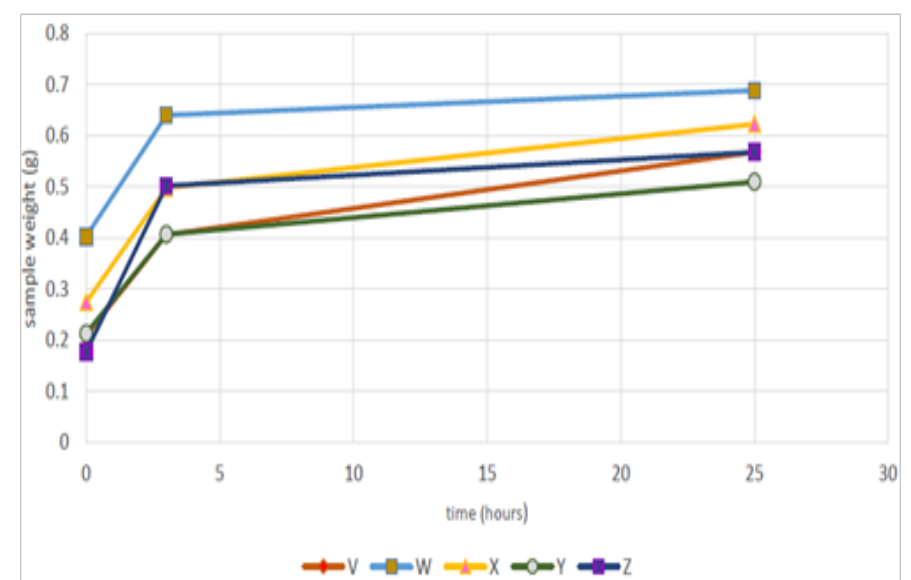

Figure 4 The steepest increase is observed for sample $Z$ which consists of the highest concentration of $\beta$-Glycerophosphate $-0.40 \mathrm{ml}$. Sample $V$ and $Y$ overlap each other for the first 3-5 hours after being immersed in PBS. This demonstrates that the rate of hydrogen bonding formation is same for both the hydrogels for the first 3-4 hours. 


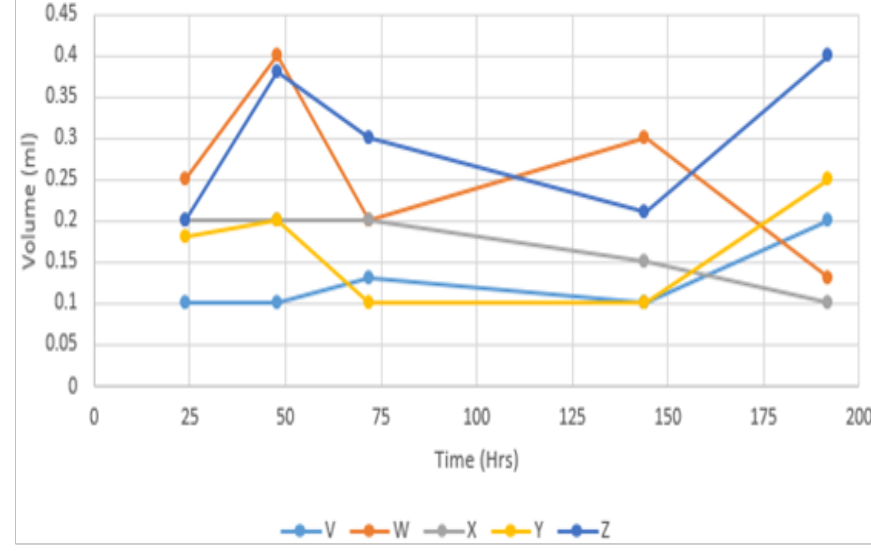

Figure 5 Graph displaying the weighed samples after being immersed in PBS solution for given time periods. Sample $X$ demonstrated poor swelling ability compared to all the other samples. For some samples, the water uptake seemed to be dependent on the chitosan proportion as the water uptake decreased with higher concentration of $\beta$-GP. Samples $V$ and $W$ showed to be less viscous compared to the other samples as they both demonstrated poor swelling capabilities.

Table 2 Recorded volume displacement for each sample in swelling test 2

\begin{tabular}{llllll}
\multicolumn{5}{l}{ Volume displacement $(\mathrm{ml})$} \\
Samples & \multicolumn{5}{l}{ Time (hours) } \\
\cline { 2 - 6 } & $\mathbf{2 4}$ & $\mathbf{4 8}$ & $\mathbf{7 2}$ & $\mathrm{I} 44$ & $\mathbf{1 9 2}$ \\
\hline $\mathrm{V}$ & 0.1 & 0.1 & 0.13 & 0.1 & 0.2 \\
$\mathrm{~W}$ & 0.25 & 0.4 & 0.2 & 0.3 & 0.13 \\
$\mathrm{X}$ & 0.2 & 0.2 & 0.2 & 0.15 & 0.1 \\
$\mathrm{Y}$ & 0.18 & 0.2 & 0.1 & 0.1 & 0.25 \\
$\mathrm{Z}$ & 0.2 & 0.38 & 0.3 & 0.21 & 0.4 \\
\hline
\end{tabular}

\section{Conclusion}

Variations in storage moduli at $1 \mathrm{Hs}$ for all five samples with different loading of the cross-linker shows that cross-linking the hydrogel with $0.20 \mathrm{ml}$ of $\beta$-GP improves its mechanical properties compared to other samples with different value of $\beta$-GP. Storage modulus for all samples showed their elastic properties. Loss modulus for all samples demonstrated their viscosity. Material has to be viscous in order to adhere to the defect site when placed in the articular cartilage. This is because articular cartilage undergoes constant pressure therefore, the material should have adhering properties. If a material has very high viscosity, it is not seen as an advantage, because it would prevent the flow of nutrients to the defect site for the healing process. ${ }^{21,22} \mathrm{~A}$ drawback of very low value for loss modulus would mean poor adhering ability of the material which would prevent the scaffold fixating at the defect site. ${ }^{23-25}$ This study shows that the hydrogels formed during the experiment have the potential to be used as scaffolds for articular cartilage. Samples Y and Z with $0.20 \mathrm{ml}$ and $0.40 \mathrm{ml}$ of $\beta$-GP demonstrated good mechanical properties when they underwent compression test. The composition of cross-linker for both samples was $0.20 \mathrm{ml}$ and $0.40 \mathrm{ml}$ respectively. The samples' viscoelastic behaviour also demonstrates good mechanical properties and such properties make hydrogels suitable for articular cartilage repair growth.
The best composition for the hydrogel is for sample $Y$ with $0.20 \mathrm{ml}$ $\beta$-GP cross-linker because it has the highest values for storage and loss modulus followed by sample $\mathrm{V}$ with $\beta$-GP concentration of $0.02 \mathrm{ml}$. This is suitable for articular cartilage because the higher the values, the better the composition of hydrogel. Articular cartilage experiences 1 to $4 \mathrm{MPa}$ of force and no synthetic material has been able to come close to withstanding such forces. ${ }^{26,27}$ For the swelling test, each hydrogel sample interacted with the water molecules. This demonstrated that the hydrogel was able to form hydrogen bonds giving variable range porosity of the material. The water content in articular cartilage is what makes it resilient to applied forces. These pores demonstrate one of the important properties for a hydrogel as the nutrients diffuse through these pores for chondrocytes to facilitate articular cartilage repair. ${ }^{28-30}$

\section{Acknowledgements}

None.

\section{Conflict of interest}

The author declares no conflict of interest.

\section{References}

1. Matricardi P, Alhaique F, Coviello T. Polysaccharide Hydrogels: Characterization and Biomedical Applications, USA: Pan Stanford Publishing; 2016.

2. Michalek J. Hydrogels in Biology and Medicine. New York, USA: Nova; 2010.

3. Peppas NA, Hoffman AS. Hydrogels. Biomaterials science. 3rd ed. Cambridge, USA: Academic Press; 2013. p. 166-179.

4. Ahmed EM. Hydrogel: preparation, characterization, and applications: A review. J Adv Res. 2015;6(2):105-121.

5. Boucard N, Viton C, Agay D, et al. The use of physical hydrogels of chitosan for skin regeneration following third-degree burns. Biomaterials. 2007;28(24):3478-3488.

6. Bullock AJ, Pickavance P, Haddow DB, et al. Development of a calcium-chelating hydrogel for treatment of superficial burns and scalds. Regen Med. 2010;5(1):55-64

7. Madaghiele M, Demitri C, Sannino A, et al. Polymeric hydrogels for burn wound care: Advanced skin wound dressings and regenerative templates. Burns Trauma. 2015;2(4):153-161.

8. Sun G, Zhang X, Shen YI, et al. Dextran hydrogel scaffolds enhance angiogenic responses and promote complete skin regeneration during burn wound healing. Proc Natl Acad Sci USA. 2011;108(52):20976-20981.

9. Flory PJ. Principles of polymer chemistry. Ithaca, New York: Cornell University Press; 1953. 688 p.

10. Ratner BD. Biomaterials science: an introduction to materials in medicine. 3rd ed. Cambridge, USA: Academic Press; 2013.

11. Yuan Y, Chesnutt BM, Haggard WO, et al. Deacetylation of chitosan: material characterization and in vitro evaluation via albumin adsorption and pre-osteoblastic cell cultures. Materials (Basel). 2011;4(12):1399-1416.

12. Kenedi RM. Perspectives in Biomedical engineering: proceedings of a symposium organised in association with the biological engineering society and held in the University of strathclyde, Glasgow, June 1972. UK: Palgrave macmillan: 1973. $314 \mathrm{p}$.

13. Espino DM, Shepherd DET, Hukins DWL. Viscoelastic properties of bovine knee joint articular cartilage: dependency on thickness and loading frequency. BMC Musculoskelet Disord. 2014;15(1):205. 
14. Noishiki Y, Takami H, Nishiyama Y, et al. Alkali-induced conversion of beta-chitin to alpha-chitin. Biomacromolecules. 2003;4(4):896-899.

15. Clar C, Cummins E, McIntyre L, et al. Clinical and cost-effectiveness of autologous chondrocyte implantation for cartilage defects in knee joints: systematic review and economic evaluation. Health Technol Assess. 2005;9(47):1-82.

16. Corpus KT, Bajaj S, Daley EL, et al. Long-term evaluation of autologous chondrocyte implantation: minimum 7-year follow-up. Cartilage. 2012;3(4):342-350.

17. Fox AJS, Bedi A, Rodeo SA. The basic science of articular cartilage: structure, composition, and function. Sports Health. 2009;1(6):461-468.

18. Frenkel SR, Di Cesare PE. Scaffolds for articular cartilage repair. Ann Biomed Eng. 2004;32(1):26-34.

19. Hutmacher DW. Scaffolds in tissue engineering bone and cartilage. Biomaterials. 2000;21(24):2529-2543.

20. James CB, Uhl TL. A review of articular cartilage pathology and the use of glucosamine sulfate. J Athl Train. 2001;36(4):413-419.

21. Sefat F. Bone Cells and Mechanical Stimulation. Scholars, Germany: Lambert Academic Publishing Press; 2013

22. Sefat F, Youseffi M, Khaghani SA, et al. Effect of Transforming Growth Factor- $\beta_{3}$ on mono and multilayer chondrocytes. Cytokine. 2016;83:118126.
23. Sefat F, Denyer MCT, Youseffi M. Effects of different transforming growth factor beta (TGF- $\beta$ ) isomers on wound closure of bone cell monolayers. Cytokine. 2014;69(1):75-86.

24. Sefat F, Khaghani SA, Kaur P, et al. Fabrication and Characterisation of Hydrogels for Cartilage Repair. Regenerative medicine and biomaterials conference: meibioeng. UK: Oxford; 2016.

25. Sefat F. Transforming growth factor beta (TGF- $\beta$ ): natural curing agents for repair. MOJ Cell Sci Report. 2014;1(3):00009.

26. Cole BJ, Gomoll A. Biologic joint reconstruction: alternatives to arthroplasty. SLACK. J Sports Sci Med. 2009;8(2):313.

27. Briggs TW, Mahroof S, David LA, et al. Histological evaluation of chondral defects after autologous chondrocyte implantation of the knee. $J$ Bone Joint Surg Br. 2003;85(7):1077-1083.

28. Leddy HA, Christensen SE, Guilak F. Microscale diffusion properties of the cartilage pericellular matrix measured using 3D scanning microphotolysis. J Biomech Eng. 2008;130(6):061002.

29. Abdeldayem A, Youseffi M, Sefat F, et al. The EFFECT of WIN55, 212-2 on protein $\mathrm{S} 100$, matrix metalloproteinase-2 and nitric oxide expression of chondrocyte monolayer. SM J Biomed Eng. 2017;3(1):1011.

30. Khaghani SA, Sefat F, Youseffi M, et al. Effect of transforming growth factor- $\beta$ on up/down regulation of integrin- $\beta 1$ in primary chondrocyte culture. European Cells and Materials. 2016;32:56. 